\title{
Внедрение сценарного подхода в разработку стратегии компании
}

\author{
Черкасова В.А. ${ }^{21}$, Фрадкина М.М. ${ }^{22}$
}

В статье представлена методика разработки стратегии компании с использованием сиенарного подхода. Для этого выделяются основные факторы, влияющие на принятие компанией стратегических решений, описываются подходы к моделированию динамики ключевых показателей и строится модель принятия решений на основе сиенарного планирования.

C проблемой принятия стратегических решений в условиях неопределенности относительно будущего сталкивается любая компания в рыночной экономике. В условиях экономического кризиса, когда уровень неопределенности увеличивается, эта проблема становится особенно актуальной. Одним из методов уменьшения влияния фактора неопределенности на деятельность компании, получивщим новый толчок к развитию во второй половине $X X$ века, является сценарной подход. Oн дает возможность проанализировать влияние возможных изменений факторов $u$ их сочетаний на деятельность компании и принять решение об адекватности стратегии развития компании и возможности ее реализащии в данной экономической ситуации.

Представленная статья предлагает одну из возможных методик сиенарного подхода применительно к реализачии стратегии компании, основанную на использовании алгоритма в рамках модели поиска сценариев, разработанной Бриантом и Лемпертом [Bryant, Lempert, 2010]. Симуляционная модель, на основе которой разрабатываются сценарии, может учитывать как внутренние стратегические переменные, так и большое количество внешних факторов, которые оказывают влияние на деятельность компании.

\section{JEL: G31}

Ключевые слова: сиенарный подход, симулячиионное моделирование, стратегия компании, регрессионный анализ, предопределенные и неопределенные переменные

Неопределенность - одна из главных характеристик окружающего мира. Каждый день компания сталкивается с огромным количеством факторов, которые влияют на ее деятельность, финансовые результаты, рыночную стоимость. Изменение множества из них невозможно точно спрогнозировать. Конечной целью, которую преследуют финансовые менеджеры, является максимизация стоимости фирмы. Важная задача - поиск ключевых факторов, которые могут влиять на деятельность компании и ее стратегические решения.

Менеджеры компании стараются выбрать наилучшую стратегию, которая улучшит финансовые показатели фирмы и усилит ее конкурентоспособность. Возможность предсказать будущие денежные потоки в соответствии с рассматриваемой стратегией может оказаться в этом случае очень полезной. Исследователи разрабатывают множество методик для того, чтобы предугадать возможные последствия принимаемых решений. Различные методики могут быть применены в зависимости от основных характеристик компании: размера, рынка, организационной структуры, предпочтений менеджмента. Один из подходов к борьбе с неопределенностью основан на построение возможных сценариев развития будущего.

Сценарный подход имеет долгую историю, которую можно проследить в работах ранних философов, например «Республике» Платона. Разработка сценариев также широко

\footnotetext{
${ }^{21}$ Канд. эконом. наук, доцент кафедры экономики и финансов фирмы ГУ ВШЭ.

${ }^{22}$ Бакалавр экономики, ГУ ВШЭ.
} 
использовалась при ведении войн [Bradfield, et al., 2005]. В XX веке идея сценарного планирования будущего стала довольно популярной. После Второй мировой войны множество статей были посвящены разработке наилучшего метода генерирования сценариев. А после того как компания Shell предсказала нефтяной кризис 1973 года, используя сценарное планирование, подход доказал свою применимость к экономическим проблемам. Исследование сценарного подхода продолжается и в настоящее время, так как для того чтобы оставаться успешной и конкурентоспособной, любая компания вынуждена тщательно просчитывать все возможные варианты развития событий и последствия принимаемых решений.

Существует множество способов разработки сценариев. В то же время при построении сценария в каждом конкретном случае должен быть выработан свой подход исходя из особенностей деятельности, управленческой структуры компании, доступности данных, возможности осуществления корректных экспертных оценок, уровня неопределенности относительно динамики факторов и экономической ситуации в целом. Применяемый метод может быть и комбинацией существующих и наиболее популярных подходов к построению сценариев. В рамках сценарного подхода можно выделить два противоположных направления, основанных на качественных и количественных методах анализа. Наиболее яркий представитель количественных методов - симуляционный, который объединяет множество математических подходов, основанных на симуляции поведения системы показателей в различных условиях. Он используется для построения сценариев, отвечающих на вопрос «что, если?».

Сценарии, основанные на симуляционном подходе, строятся в несколько этапов: непосредственная разработка модели; сбор данных; тестирование модели с использованием данных; анализ сценариев. Преимущества данного метода состоят в том, что модель легко проверить на имеющихся данных. Тем не менее данный метод является достаточно дорогим и трудоемким и требует хорошей и достоверной базы данных. Однако он может задействовать меньшее количество людей, чем другие методы. Также позволяет рассматривать как альтернативные сценарии, так и экстраполировать существующие тренды в зависимости от входных параметров модели.

Применение этого метода требует тщательной подготовки перед непосредственной разработкой сценариев. Важно заранее проанализировать деятельность компании, выделить в ней основные направления и факторы, которые оказывают влияние на ее работу. Все обстоятельства, оказывающие влияние на деятельность компании, ее финансовый результат и стоимость, можно разделить на три группы [Van Notten et al., 2003].

Первая группа - это действующие лица (actors). Кроме самой компании, эта группа включает в себя компании-конкуренты, организации потребителей, государственные органы, то есть экономические агенты, существующие в данной экономической системе и прямо или косвенно взаимодействующие с данной фирмой.

Вторую группу можно условно назвать факторами (factors). K ним относятся различные макро- и микроэкономические показатели, такие как занятость, потребление, цены, уровень цен в экономике и т.д. Все факторы можно разделить на две группы. Внутренние факторы контролируются рассматриваемым действующим лицом, тогда как внешние факторы находятся за пределами его зоны влияния [Borjeson et al., 2006]. Таким образом, компания управляет внутренними факторами на основе принимаемых стратегических решений, например на прогнозе динамики внешних факторов, которые можно разделить на несколько групп в зависимости от того, насколько предопределено их поведение [Postma et al., 2005]. Первая группа - постоянные (неизменные) факторы. К ним относят различные структурные показатели, которые не могут значительно меняться в пределах видимого временного горизонта. Например: налоговые ставки, государственная структура, уровень институционального развития. Предопределенные факторы можно предсказать с достаточной степенью уверенности. По ним собрана значительная статистика, известны вероятности тех или иных событий или выделен тренд, объясняющий 
значительную часть их поведения. Как правило, они связаны с демографическими и социальными показателями. Для компании это могут быть объем производства, отпускные цены, издержки на производство.

Неопределенные факторы не имеют никакой закономерности, или закон, определяющий их значение, неизвестен, и их поведение является основной причиной различий в сценариях будущего. Это форс-мажорные обстоятельства, шоки спроса и предложения как на товары, так и на ресурсы.

Факторы, которые являются внешними для рассматриваемой компании, могут быть внутренними для других действующих лиц. Например, это относится к законодательным нормам, ценовой политике конкурентов или валютному курсу при режиме фиксированного валютного курса. Другие факторы (например, уровень цен в ситуации, близкой к совершенной конкуренции, или фондовые индексы) являются внешними для всех участников. Вопрос о том, для кого какой фактор является внешним или внутренним, в каждом конкретном случае должен решаться отдельно.

Также следует учитывать, что значения ряда факторов могут определяться не напрямую, а косвенно, через значения других переменных.

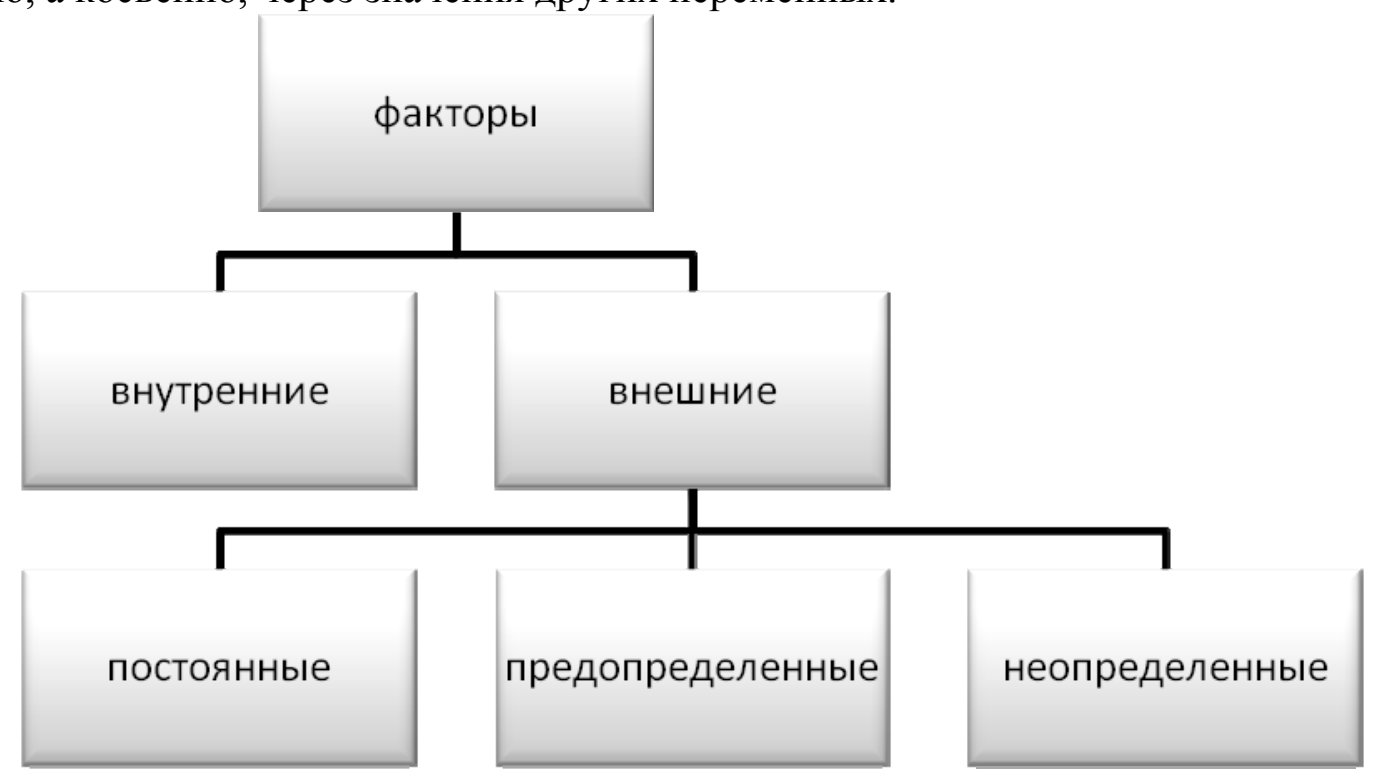

Третью группу обычно называют секторами (sectors). Это те арены и рынки, на которых взаимодействуют действующие лица и факторы.

Разработка любого сценария должна начинаться с определения существующего положения дел, то есть сектора экономики, в котором ведет свою деятельность компания, основных действующих лиц и факторов, определяющих деятельность компании.

Построение системы, в которой ведет свою деятельность компания, как правило, является сложной и нетривиальной задачей. Но она может быть упрощена при условии выделения ключевых областей деятельности компании и их независимом анализе или целевых показателей, которые будут определять всю дальнейшую деятельность компании.

После составления системы показателей становится очевидным тот факт, что для моделирования возможных вариантов динамики как внешних, так и внутренних факторов требуется применение математического и статистического аппарата. Первым этапом становится подбор модели, которая количественно свяжет все значимые переменные.

Когда внутри данной системы существуют какие-либо причинно-следственные связи, для которых могут быть подобраны соответствующие уравнения, полезным может оказаться методика, основанная на объяснительном моделировании (explanatory modeling). Основной целью построения таких моделей является тестирование гипотез о существовании связей. В случае успешного их нахождения, задавая различную динамику переменных или набора переменных, можно получить различные сценарии будущего. Негативный момент состоит в том, что неточное определение причинно-следственных связей или изменение их природы 
может приводить к искажениям результатов анализа. Однако если уравнения не выводятся математически, а моделируются с помощью регрессионного анализа, стоит помнить, что не всегда чем выше объясняющая сила модели, тем выше ее предсказательная сила. Объясняющая сила модели тем выше, чем меньше:

(1) Bias $=\left[f^{*}(X)-f(X)\right]^{2}$,

где $E(Y)=f(X)$ - истинная модель,

$E(Y)=f^{*}(X)-$ недоопределенная модель.

Предсказательная сила модели тем выше, чем меньше:

$$
M S E=E(Y-\hat{Y} \mid x)^{2}=\operatorname{Var}(Y)+\operatorname{Bias}^{2}+\operatorname{Var}\left(\hat{f}^{*}(x)\right),
$$

где $\hat{f}^{*}(X)$ - оцененная недоопределенная модель.

В некоторых случаях неправильно определенная модель обладает большей предсказательной силой, чем истинная.

Это происходит в следующих случаях:

1) когда существует высокая дисперсия остатков;

2) если истинные абсолютные значения коэффициентов малы;

3) если объясняющие переменные коррелируют (мультиколлинеарность);

4) когда существуют маленькая выборка или небольшой разброс значений (range) объясняющих переменных, которые не включаются в недоопределенную модель.

Проиллюстрировать это можно на примере истинной модели следующего вида:

$$
f(x)=\beta_{1} x_{1}+\beta_{2} x_{2}+\varepsilon, \text { где } x=\left[x_{1} ; x_{2}\right], \operatorname{Var}(\varepsilon)=\sigma^{2} .
$$

Если модель правильно определена, то:

$$
\operatorname{Var}\left(\hat{\beta}_{1} x_{1}+\hat{\beta}_{2} x_{2}\right)=\sigma^{2} x^{\prime}\left(X^{\prime} X\right)^{-1} x
$$

$$
\begin{aligned}
& \text { Bias }=0 \\
& M S E_{1}=E(Y-f(x))^{2}=\sigma^{2}+0+\operatorname{Var}\left(\hat{\beta}_{1} x_{1}+\hat{\beta}_{2} x_{2}\right)=\sigma^{2}\left[1+x^{\prime}\left(X^{\prime} X\right)^{-1} x\right] .
\end{aligned}
$$

Если модель недоопределена, то уравнение принимает вид:

$$
\begin{aligned}
& \hat{f}^{*}(x)=\hat{\gamma}_{1} x_{1} \\
& \operatorname{Bias}=\gamma_{1} x_{1}-\left(\beta_{1} x_{1}+\beta_{2} x_{2}\right)=x_{1}\left(x_{1}{ }^{\prime} x_{1}\right)^{-1} x_{1} \\
& \operatorname{Var}\left(\hat{\gamma}_{1} x_{1}\right)=\sigma x_{1}\left(x_{1}{ }^{\prime} x_{1}\right)^{-1} x_{1} \\
& \operatorname{MSE}_{2}=E\left(y-\hat{f}^{*}(x)\right)^{2}=\left(x_{1}\left(x_{1}{ }^{\prime} x_{1}\right)^{-1} x_{1}{ }^{\prime} x_{2} \beta_{2}-x_{2} \beta_{2}\right)^{2}+\sigma^{2}\left[1+x_{1}\left(x_{1}{ }^{\prime} x_{1}\right)^{-1} x_{1}\right] .
\end{aligned}
$$

При сравнении $M S E_{1}$ и $M S E_{2}$ видно, что в некоторых случаях прогнозная сила неправильно определенной модели может оказаться выше, несмотря на то что объясняющая сила всегда выше у правильно определенной модели.

Таким образом, для целей сценарного планирования требуется выбирать модель с наибольшей предсказательной силой.

Следующим вопросом является моделирование динамики независимых переменных. Можно смоделировать поведение переменных на основе экстраполяции данных при помощи анализа временных рядов [Harries, 2003]. Такой способ используется, если динамика переменных в основном определена их предыдущими значениями или не влияет значительно на результирующие показатели. Главный минус этого метода состоит в том, что природа будущих изменений может значительно отличаться от тех, которые наблюдались в прошлом. Этот метод может применяться для предопределенных переменных.

Если уровень неопределенности высок и фактор не показывает никакой связи с другими, для генерирования его значений наиболее удобным способом может оказаться поиск закона распределения. Одним из самых простых способов является аппроксимация закона распределения в выборке на основе типовых распределений. Этот метод состоит из нескольких этапов: 
- предварительный выбор закона распределения на основе визуального анализа гистограмм выборки, коэффициентов распределения и эксцесса;

- определение оценок параметров закона распределения;

- оценка соответствия закона распределения исходным данным.

Одним из способов генерирования большого количества сценариев является метод симуляций Монте-Карло, который состоит в генерировании сценариев в соответствии со специфической функцией распределения. Исследуемый процесс моделируется путем многократных повторений его случайных реализаций. Единичные реализации называются статистическими испытаниями. Таким образом, можно генерировать бесконечное количество сценариев или групп сценариев в соответствии с заданным законом распределения неопределенных переменных. Критический момент состоит в том, что требуется обосновать выбор функции распределения, которая наиболее полно отражает природу факторов. Наиболее часто используемая функция распределения - это многомерное стандартное нормальное распределение $\mathrm{N}(0, \mathrm{In})$ с нулевым математическим ожиданием и единичной ковариационной матрицей In. [Guastariba and Mansini 2009].

Следующим этапом после выбора симуляционной модели и моделирования динамики основных показателей становится непосредственная разработка сценариев. Разработка сценария в каждом конкретном случае - процесс творческий. Однако для конкретных требований, применяемых к результату проекта, можно выделить определенные требования и к сценариям. В данной статье рассмотрим методику моделирования сценариев, отвечающих следующим требованиям:

- у учет влияния всех выявленных переменных на результаты проекта;

- возможность учета взаимосвязей между внутренними факторами;

- возможность объединить возможные сценарии в несколько групп в зависимости от значения результирующего показателя;

- моделирование сценариев исключительно на основе доступных количественных данных;

- возможность моделировать сценарии в зависимости от предлагаемых стратегических решений.

За основу предлагаемой методики разработки сценариев и влияющей на выбор компанией определенного стратегического решения взят подход, предложенный Бриантом и Лемпертом [Bryant and Lempert, 2010], основанный на принятии разумных решений (robust decision making). В общем случае он дает возможность получить сценарии, показывающие слабые места предлагаемой политики. В данной статье методику модифицируем для анализа стратегических решений компании.

Первый этап предполагает наличие количественной взаимосвязи между ключевыми переменными. Для этого используем одну или несколько симуляционных моделей следующего вида:

(6)

$$
y=f(s, x),
$$

где $s$ - вектор значений управляемой переменной,

$y$ - значение целевой функции,

$x$ - вектор внешних переменных, значения которых могут задаваться законом распределения.

Усложним данную модель, включив в нее фактор времени и взаимосвязи между факторами. Теперь модель может быть представлена в следующем виде:

$$
y=f\left(s_{1} \ldots s_{n}, x_{1} \ldots x_{n}, b_{1} \ldots b_{n}, c\right),
$$

где $s$ - вектор значений управляемой (внутренней переменной),

$x$ - вектор значений неопределенных факторов,

$b$ - вектор предопределенных факторов.

Значение величины в каждый момент времени может задаваться следующим образом: 
(8)

$$
b_{t}=\alpha_{0}+\alpha_{1} b_{t-1}+\ldots+\alpha_{n} b_{t-n}+\beta_{1} x_{t-1}+\ldots+\beta_{m} x_{t-m}+\gamma c
$$

где $c$ - вектор постоянных факторов.

Важным вопросом является выбор $y$, как стратегической целевой переменной. Это может быть стоимость компании, доля рынка, объем продаж или любая другая переменная, которую можно рассматривать в качестве стратегической цели компании. В качестве результирующего показателя эффективности проекта может выбираться его чистая приведенная стоимость (NPV), так как данный показатель позволяет учитывать эффективность инвестиций в проект за весь период реализации, в отличие от таких показателей, как NOPAT (чистая операционная прибыль) и EBITDA (прибыль до уплаты процентов, налога на прибыль и амортизации), которые иллюстрируют деятельность компании за период.

После того как построена симуляционная модель, следует определить область значений переменной $y$, для того чтобы отсечь изначально неправдоподобные варианты развития событий. Так, доля рынка ограничена значениями от 0 до 1. Разделив область значений $y$ на несколько частей, можно проанализировать, какие значения неопределенных факторов приведут к тем или иным результатам. Предлагается первичным критерием для выделения комплектов сценариев выбрать благоприятность значения целевого показателя, выделяя три комплекта сценариев и отсекая изначально неприемлемые результаты, такие как отрицательное значение приведенной стоимости.

Таким образом, выделяется область значений целевой переменной, представляющая интерес. Выбирается критерий Y', который определяет верхнюю или нижнюю границу переменной:

$$
I_{s}=\left\{x^{\prime} \mid f\left(s, x^{\prime}\right) \geq Y^{\prime}\right\} \text { или }\left\{x^{\prime} \mid f\left(s, x^{\prime}\right) \leq Y^{\prime}\right\} .
$$

После чего с помощью симуляции Монте-Карло получают сочетания переменных:

$$
\left\{y_{i}, x_{i} \mid b_{i}, c_{i}\right\} \text {. }
$$

Если целевым показателем является, например, NPV, то генерируются случайным образом сочетания параметров (в качестве математического ожидания берется значение параметра на дату проведения анализа) и определяется конечное значение NPV для каждого из этих случаев. В данном примере главная цель - охватить максимальное количество возможных сочетаний параметров для того, чтобы найти границу между теми сценариями, которые приведут к положительным или отрицательным значениям приведенной стоимости. В качестве области значений $I s$, представляющей интерес, выбираются значения NPV $>0$.

Второй этап - определение возможных сценариев, которые наилучшим образом предсказывают область значений целевой переменной Is. Для этого используем ограничения следующего вида:

(11) $B_{k}=\left\{a_{j} \leq x_{j} \leq b_{j}, j \in L_{k}\right\}$,

где $L_{k} \subseteq\{1, \ldots, M\}$. Область определения остальных переменных не ограничена. Каждое ограничение $B_{k}$ интерпретируется как сценарий.

Выделим набор сценариев, дающих возможность проанализировать стратегическую альтернативу наилучшим образом. Для того чтобы грамотно определить границы сценариев, используем три показателя, часто противоречащие друг другу, которые могут измерить уровень сценариев:

$$
\begin{aligned}
& \text { Покрытие }=\sum_{x_{i}=B} y_{i}{ }^{\prime} / \sum_{x_{i} \in x^{\prime}} y_{i}{ }^{\prime}, \\
& \text { Концентращия }=\sum_{x_{i} \in B} y_{i}{ }^{\prime} / \sum_{x_{i} \in B} 1,
\end{aligned}
$$

где $y_{i}{ }^{\prime}=1$, если $-x_{i} \in I_{s}, y^{\prime}=0-$ влюбом другом случае

Интерпретируемость - наличие экономического обоснования получившихся результатов. 
Наилучшим результатом моделирования сценариев будет учет значительной доли значений у, относящихся к интересующей компанию области значений целевой переменной (покрытие), минимизация количества значений у в сценарии, не относящихся к области значений, представляющей интерес (высокая концентрация) и высокая интерпретируемость. Поскольку эти ограничения редко выполняются одновременно, может потребоваться пожертвовать одним показателем за счет другого, например уменьшая количество переменных для лучшей интерпретируемости. Для выбора наилучшего соотношения между критериями предлагается использовать алгоритм, основанный на визуализации коэффициентов покрытия и концентрации. Используя два из вышеперечисленных критериев - покрытие и концентрацию, можно построить кривые, иллюстрирующие альтернативные значения коэффициентов при различном количестве факторов, на значения которых накладываются ограничения при построении сценариев. Чем меньше ограничений - тем легче интерпретировать сценарий. В зависимости от требований к проекту выбирается набор сценариев.

На третьем этапе проводится диагностика сценариев. То есть проверка того, насколько корректно изменения результирующей величины реагируют на малые изменения параметров. Для этого могут использоваться разные тесты. Например, тест повторной выборки (resampling test) проверяет частоту, с которой могут получаться одинаковые значения целевой переменной при фиксированной базе данных. Процедура предполагает «проверку алгоритма на множестве подвыборок из базы данных и выявляет, какие из ограничений на параметры постоянно оказывают влияние на значения результирующей величины» [Bryant, Lempert, 2010].

Квази пи-вэлью тест (quasi p-value test) тестирует, не привело ли применение алгоритма к искажению результатов. Процедура описывается следующим образом.

Рассматривается сценарий $\beta$ из набора сценариев $B$ с ограничениями на параметры $L \beta$ и сценарий $\beta_{-j}$ с ограничениями на все переменные, кроме $x_{j}$. Первый сценарий предполагает реализацию одного из $H$ значений переменной $y$, попадающих в $I_{s}$, из $T$. А второй $-H_{-j}$ и $T_{-j}$ соответственно: $T_{-j} \geq T, H_{-j} \geq H$. Основная гипотеза: реализации $y$ из $I_{S}$ в $\beta_{-j}$ распределены среди всех реализаций $y$ в соответствии с законом распределения: $p(1)=\frac{H_{-j}}{T_{-j}}$.

На основании результатов этих тестов принимается решение об адекватности данных сценариев. После завершения моделирования сценариев принимается решение о приемлемости данной стратегии для компании или проведении анализа для альтернативных решений.

Данный алгоритм дает возможность построить набор сценариев, соответствующий стратегической альтернативе, выделить ключевые факторы, которые наиболее сильно влияют на результирующий показатель. Механизм моделирования сценариев построен таким образом, что, несмотря на использование количественных методов анализа, позволяет разрабатывать сценарии, поддающиеся интерпретации с экономической точки зрения.

Таким образом, данная методика позволяет учитывать влияние множества факторов и их взаимосвязей на целевой показатель, выбранный в качестве индикатора успешности реализации стратегии. Симуляционная модель, на основе которой разрабатываются сценарии, может учитывать как внутренние стратегические переменные, так и большое количество внешних факторов, которые оказывают влияние на деятельность компании. Методика предполагает выбор одного из наборов сценариев, обладающих приемлемыми значениями коэффициентов покрытия, концентрации и интерпретируемости, иллюстрирующих те сочетания значений факторов, которые приведут к успешной реализации стратегии. На основе такого анализа представляется возможным сделать выводы о правдоподобности данных исходов и возможности реализации стратегии. В случае если уровень риска при принятии данного стратегического решения признается неприемлемым, 
могут быть рассмотрены альтернативные решения и проанализированы условия для их успешной реализации.

\section{Список литературы}

1. Börjeson, L., Höjer, M., Dreborg, K.-H., Ekvall, T., Finnveden, G. (2006), Scenario types and techniques: towards a user's guide, Futures, 38(7) (2006) 723-739

2. Bradfield, R., Wright, G., Burt, G., Cairns, G., Van der Heijden, K. (2005), The origins and evaluation of scenario techniques in long range business planning, Futures, 37 (2005) 795812.

3. Bryant, B.P., Lempert, R.J. (2010), Thinking inside the box: A participatory, computer assisted approach to scenario discovery, Technological Forecasting \& Social Change, 77 (2010) 34-49.

4. Chichester, J.W., Sons. van Notten, P. W. F., and Rotmans, J. (2003), An updated scenario typology, Futures, 35(5) (2003) 423-443.

5. Guastaroba, G., Mansini, R., Speranza, M.G. (2009), On the effectiveness of scenario generation techniques in singe-period portfolio optimization, European Journal of Operational Research, 192 (2009) 500-511.

6. Harries, Z.C. (2003), Correspondence to what? coherence to what? what is good scenariobased decision making? Technological Forecasting and Social Change, 70 (2003) 797-817.

7. Postma, T.J.B.M., Liebl, F. (2005), How to improve scenario analysis as a strategic management tool?, Technological Forecasting \& Social Change, 72 (2005) 161-173. 